\title{
Tailored antihypertensive drug therapy prescribed to older women attenuates circulating levels of interleukin- 6 and tumor necrosis factor- $\alpha$
}

This article was published in the following Dove Press journal:

Clinical Interventions in Aging

9 January 2015

Number of times this article has been viewed

\author{
Juliana O Toledo' \\ Clayton F Moraes ${ }^{2,3}$ \\ Vinícius C Souza ${ }^{2}$ \\ Audrey C Tonet-Furioso ${ }^{2}$ \\ Luís CC Afonso ${ }^{4}$ \\ Cláudio Córdova ${ }^{3}$ \\ Otávio T Nóbregal,2 \\ 'Graduate Program in Health Sciences, \\ ${ }^{2}$ Graduate Program in Medical Sciences, \\ University of Brasília, Brasília, ${ }^{3} \mathrm{Graduate}$ \\ Program in Gerontology, Catholic \\ University of Brasília, Brasília, ${ }^{4}$ Research \\ Center in Biological Sciences, Federal \\ University of Ouro Preto, Ouro Preto, \\ Brazil
}

Objective: To test the hypothesis that antihypertensive drug therapy produces anti-inflammatory effects in clinical practice, this study investigated circulating levels of selected proinflammatory mediators (interleukin-6 [IL-6], tumor necrosis factor-alpha [TNF- $\alpha$ ], and interferon- $\gamma$ [INF- $\gamma]$ ) in response to multivariate drug directions for blood pressure (BP) control.

Methods: Prospective study involving 110 hypertensive, community-dwelling older women with different metabolic disorders. A short-term BP-lowering drug therapy was conducted according to current Brazilian guidelines on hypertension, and basal cytokine levels were measured before and after intervention.

Results: Interventions were found to represent current hypertension-management practices in Brazil and corresponded to a significant reduction in systolic and diastolic BP levels in a wholegroup analysis, as well as when users and nonusers of the most common therapeutic classes were considered separately. Considering all patients, mean IL- 6 and TNF- $\alpha$ levels showed a significant decrease in circulating concentrations $(P<0.01)$ at the endpoint compared with baseline, whereas the mean INF- $\gamma$ level was not significantly different from baseline values. In separate analyses, only users of antagonists of the renin-angiotensin system and users of diuretics exhibited the same significant treatment-induced reduction in serum IL- 6 and TNF- $\alpha$ observed in the whole group.

Conclusion: Our data demonstrates that a clinically guided antihypertensive treatment is effective in reversing the low-grade proinflammatory state of serum cytokines found in postmenopausal women and support extracardiac benefits from diuretics and renin-angiotensin system antagonists.

Keywords: renin-angiotensin system, cytokine, hypertension, drug therapy, guidelines, inflammation

\section{Introduction}

Angiotensin II, the active principle of the renin-angiotensin system (RAS), in addition to playing a role in the regulation of blood pressure (BP), shows key proinflammatory effects upon stimulation of its type 1 receptor $\left(\mathrm{AT}_{1} \mathrm{R}\right) .{ }^{1,2}$ Furthermore, sympathoexcitation and its catecholamine products also exhibit proinflammatory effects attributable to $\beta 1$-adrenoceptor-triggered pathways. ${ }^{3}$ Thus, enhanced sympathetic and RAS activities, often present in hypertension and diabetes, are important determinants of endothelial dysfunction and complications concerning the contribution of immune mediators. ${ }^{4,5}$ RAS antagonists have proven to reverse both systemic ${ }^{6}$ and site-specific (hepatic, ${ }^{7}$ lung, ${ }^{8}$ and vascular, ${ }^{9}$ among others) inflammatory phenotypes in in vivo and in vitro models. Antiinflammatory effects of these antagonists include blocking the $\mathrm{AT}_{1} \mathrm{R}$-mediated activation of nuclear factor kappa B, a transcriptional activator of proinflammatory cytokines,
Nóbrega

Programa de Pós-Graduação Stricto Sensu em Ciências Médicas, Universidade de Brasília - UnB, Campus Universitário Darcy Ribeiro, Asa Norte, Brasília, DF 70910-900, Brazil

Tel +55 6I 33072520

Email nobrega@pq.cnpq.br 
such as interleukin-6 (IL-6) and tumor necrosis factor-alpha (TNF- $\alpha$ ), and/or upregulation of its inhibitor, IкB. ${ }^{10}$ Therefore, selective $\beta 1$-adrenoceptor blockers result in suppression of norepinephrine-triggered inflammation and preferential $\beta 2$-adrenoceptor stimulation by endogenous epinephrine, with reduction in proinflammatory cytokine release. ${ }^{11-13}$

However, the clinical importance of these findings should be assessed with caution. Studies with human subjects are usually designed to produce conclusions from standardized, monotherapeutical procedures to all patients and do not usually follow the paradigm of clinical care, in which a tailored drug therapy takes into account a constellation of comorbidities, risk factors, target organ injuries, and adverse drug reactions in addition to BP levels. Concerning the elderly population, although investigations under standard conditions may provide valuable guidance to assist patients in regular circumstances, these assessments often neglect important interactions that may yield results at odds with experimental data. Our perspective relies on the assumption that an unorthodox test employing distinct, customized antihypertensive therapies based on a modern health-management policy would help test how effectively changes in the inflammatory profile could be observed in clinical practice.

In view of the foregoing and considering that the aging process is characterized by latent, overactive innate immune elements that contribute to a number of age-associated diseases, ${ }^{14}$ we examined the response of circulating cytokine levels to short-term, customized antihypertensive pharmacotherapy, following current directives on treatment of systemic arterial hypertension, prescribed to a subset of the Prognosis and Therapeutics in Geriatrics Project from Brasília, Brazil.

\section{Materials and methods}

\section{Study and subjects}

A prospective study was conducted using data obtained from community-dwelling, female outpatients from the urban outskirts of Brasília, the city capital of Brazil, aged 60 years or older and enrolled in a Catholic University of Brasília project to undertake screening and intervention to prevent atherosclerotic disorders. Our protocols were approved by the Institutional Review Board of the Catholic University of Brasília. Participation was voluntary, and written informed consent was obtained from all participants in accordance with the Declaration of Helsinki.

\section{Admission procedures}

For admission to this study, each subject was required to undergo an initial evaluation of biochemical, anthropometric, and clinical parameters. Hypertension was defined according to the IV Brazilian Guidelines on the disease, ${ }^{15,16}$ which do not deviate from international standards. Only hypertensive patients diagnosed in 2005, confirmed in 2006/2007 and who did not benefit from BP lowering with prior antihypertensive therapies were included. BP was measured after a resting period of 10 minutes, and values for each subject were the mean of at least two physician-obtained assessments. For the present investigation, held in 2009, the initial BP assessment was performed in both upper limbs, and if a difference was noticed, the higher-pressure arm was used for obtainment of all future BP measures. Patients with confirmed systemic hypertension were eligible for the study. Prior or current use of antihypertensive medication on admission accounted to the diagnostic procedure.

Body mass index (BMI; kg/m²) and waist-to-hip circumference ratio (centimeters of waist/centimeters of hip) was assessed for each patient. Body weight was measured to the nearest $0.1 \mathrm{~kg}$, with participants wearing light clothes, and height to the nearest $0.1 \mathrm{~cm}$ without shoes. Waist circumference was measured midway between the lower costal margin and iliac crest, and hip circumference was measured at the maximum width over the greater trochanters.

Laboratory evaluation included serum renal (uric acid and creatinine) and liver (aspartate aminotransferase, alanine aminotransaminase, and alkaline phosphatase) tests. Creatinine clearance was estimated using serum creatinine as described by Cockcroft and Gault. ${ }^{17}$ Fasting serum levels of glucose and lipids (triglycerides, total cholesterol, and high-density lipoprotein cholesterol) were also obtained as metabolic markers. Low-density lipoprotein cholesterol was calculated according to Friedewald's formula. ${ }^{18}$ All tests were performed in the same laboratory certified by the Accreditation Program of Clinical Laboratories of the Brazilian Society of Clinical Pathology, with reagents from Boehringer Mannheim (Germany). Serum levels of sodium and potassium were determined by flame photometry using ion-specific probes (Celm ${ }^{\circledR}$; Barueri, SP, Brazil). Proteinuria was determined by an immunonephelometric assay (Bering ${ }^{\circledR}$; Marburg, Germany) using a 24-hour urine collection.

Cases of secondary or malignant hypertension as well as clinical history of arrhythmia, coronary disease, or stroke within the previous 12 months were excluded. Other exclusion criteria were serum levels of kidney or liver markers compatible with abnormal organ function, uncontrolled type 2 diabetes mellitus and/or signs of current inflammation, infection, or malignancies. Active type 2 diabetes mellitus 
was characterized by fasting blood glucose $\geq 126 \mathrm{mg} / \mathrm{dL}$ despite the use of insulin or oral antidiabetes drugs.

\section{Follow-up clinical procedures}

The pharmacological intervention was freely chosen by the physician within the boundaries of the IV Brazilian Guidelines on Systemic Arterial Hypertension, which recommend that patients receive maintenance treatment with single or combined drug therapy based on the following drug classes: diuretics, beta blockers, angiotensin-converting enzyme inhibitors, and calcium-channel and $\mathrm{AT}_{1} \mathrm{R}$ antagonists. Each drug therapy preferably started with the minimum effective daily dose regimen, except for when a prior, ineffective regimen was already in the clinical records of the patient. Two weeks were required before increasing any dose, substituting the active principle, or switching to a combined drug therapy. Whenever clinically appropriate, diuretics were introduced as first-line treatment, followed by angiotensin-converting enzyme inhibitors or $\mathrm{AT}_{1} \mathrm{R}$ antagonists, herein referred to as RAS antagonists. All treatments were intended to be orally effective, to preserve the patient's quality of life, and to consider socioeconomic and health conditions. Instructions on how to obtain drugs from the public health system were constantly provided.

For this study, the endpoint for clinical intervention was when any reduction in systolic BP was observed between baseline and a minimum of two consecutive visits under any dosage regimen. All interventions were conducted in a blinded fashion by the practitioners.

\section{Cytokine quantification}

For the measurement of immune mediators, whole-blood samples were drawn in the morning (between 8:00 and 9:30 am) into endotoxin-free tubes at two time points: on admission to the protocol and at the endpoint of treatment. Serum was separated from clotted whole blood within 1 hour of collection and stored in aliquots at $-80^{\circ} \mathrm{C}$ until the processing day, when samples were analyzed in batches using specific enzyme-linked immunosorbent assay kits (eBioscience, San Diego, CA, USA) and processed in duplicate. The minimum detectable doses were experimentally determined as $1.0 \mathrm{pg} / \mathrm{mL}, 1.6 \mathrm{pg} / \mathrm{mL}$, and $2.0 \mathrm{pg} / \mathrm{mL}$ for IL- 6, TNF- $\alpha$, and IFN- $\gamma$, respectively. The measurements of all cytokines resulted in mean intra-assay coefficients of variation below $5 \%$.

\section{Statistical procedures}

The Kolmogorov-Smirnov test was employed to assess normal distribution of data from continuous variables in a whole-sample or in a within-group approach. The paired Student's $t$-test was used to compare the means of normally distributed variables, whereas the two-related-samples Wilcoxon test was used to compare the means for nonnormal distribution across baseline and endpoint. For nonparametric purposes, the chi-square test was used. All analyses were performed using the Statistical Package for the Social Sciences $\left(\right.$ SPSS $^{\circledR}$ ) for Windows (version 17.0). A $P$-value $<0.05$ was considered statistically significant.

\section{Results \\ Clinical outcomes}

A total of 110 patients completed the study. The baseline characteristics of the patients are summarized in Table 1 and are consistent with those expected for an elderly hypertensive sample. Mean BMI was $28.4 \mathrm{~kg} / \mathrm{m}^{2}$, which is within the clinical range for overweight $\left(27-30 \mathrm{~kg} / \mathrm{m}^{2}\right)$. Mean fasting triglyceride and glucose levels were $150.3 \mathrm{mg} / \mathrm{dL}$ and $102.3 \mathrm{mg} / \mathrm{dL}$, respectively, indicating an increased prevalence of metabolic syndrome according to worldwide consensus. ${ }^{19}$ Conversely, mean levels of all serum renal and liver functional markers were within the normal range. Table 1 also shows a remarkable degree of homogeneity in the sample regarding clinical

Table I Clinical and biochemical data of the study population

\begin{tabular}{ll}
\hline Parameters & Subjects $(\mathbf{n}=\mathbf{I ~ I 0 )}$ \\
\hline Age $($ years $)$ & $68.1 \pm 6.0$ \\
BMI $\left(\mathrm{kg} / \mathrm{m}^{2}\right)$ & $28.4 \pm 4.5$ \\
Waist-to-hip ratio & $0.87 \pm 0.06$ \\
Fasting glucose $(\mathrm{mg} / \mathrm{dL})$ & $102.3 \pm 14.0$ \\
Total cholesterol $(\mathrm{mg} / \mathrm{dL})$ & $217.4 \pm 40.6$ \\
HDL cholesterol $(\mathrm{mg} / \mathrm{dL})$ & $55.0 \pm 8.7$ \\
LDL cholesterol $(\mathrm{mg} / \mathrm{dL})$ & $132.2 \pm 37.8$ \\
Triglycerides $(\mathrm{mg} / \mathrm{dL})$ & $150.3 \pm 64.6$ \\
AST $(\mathrm{U} / \mathrm{L})$ & $24.0 \pm 7.3$ \\
ALT $(\mathrm{U} / \mathrm{L})$ & $19.1 \pm 9.1$ \\
ALP $(\mathrm{U} / \mathrm{L})$ & $176.4 \pm 63.9$ \\
Uric acid $(\mathrm{mg} / \mathrm{dL})$ & $3.71 \pm 1.01$ \\
Creatinine clearance $(\mathrm{mL} / \mathrm{min})$ & $74.8 \pm 21.3$ \\
Proteinuria $(\mathrm{mg} / 24 \mathrm{~h})$ & $144.1 \pm 66.8$ \\
Serum sodium $(\mathrm{mEq} / \mathrm{L})$ & $140.1 \pm 2.1$ \\
Serum potassium $(\mathrm{mEq} / \mathrm{L})$ & $4.29 \pm 0.42$ \\
Baseline SBP $(\mathrm{mmHg})$ & $163.0 \pm 20.6$ \\
Baseline DBP $(\mathrm{mmHg})$ & $92.5 \pm 12.2$ \\
Endpoint SBP $(\mathrm{mmHg})$ & $140.9 \pm 15.4$ \\
Endpoint DBP $(\mathrm{mmHg})$ & $83.7 \pm 8.5$ \\
DM2 & 12.7 \\
\hline Note Dat exprssd & 12.7
\end{tabular}

Note: Data expressed as mean \pm standard deviation, except for DM2 (as percentage). Abbreviations: ALP, alkaline phosphatase; ALT, alanine aminotransaminase; AST, aspartate aminotransferase; BMI, body mass index; DBP, diastolic blood pressure; DM2, type 2 diabetes mellitus; HDL, high-density lipoprotein; LDL, low-density lipoprotein; $n$, number; SBP, systolic blood pressure. 
and laboratory characteristics, expressed as low skewness values $(<3.0)$ of the continuous variables.

During drug therapy, the study subjects had an absolute count of 189 episodes of drug prescription (each individual agent considered as one episode) throughout 430 medical appointments $(2.5 \pm 1.6$ appointments/patient). The highest modal frequencies of appointments were two and three returning visits, reaching up to eight returns in a few cases. The number of prescription episodes to achieve BP reduction ranged from one to four different antihypertensive agents/ patient per day ( $1.7 \pm 0.9$ prescription episodes/patient). While $45.4 \%(n=50)$ of the users received a prescription for one single drug, 39.1\% $(n=43)$ received two and $15.5 \%(n=17)$ received three or four medications simultaneously. In our study, diuretics and RAS antagonists were the most common therapeutic classes, being used by $63.6 \%(n=70)$ and $55.5 \%$ $(\mathrm{n}=61)$ of the patients, followed by calcium-channel blockers $(24.5 \% ; n=27)$ and $\beta$-adrenergic blockers $(20 \% ; n=22)$. Between baseline and endpoint, mean systolic BP decreased $22.1 \mathrm{mmHg}(P<0.001)$ and mean diastolic BP decreased $8.8 \mathrm{mmHg}(P<0.001)$ (Table 1$)$.

\section{Association of cytokine levels with antihypertensive response}

Pretreatment and posttreatment basal cytokine levels are summarized in Figure 1. At endpoint, mean IL-6 and TNF- $\alpha$ levels showed a significant decrease in circulating concentration $(P<0.01)$ considering all patients. IFN- $\gamma$ levels at endpoint were not different from baseline. Due to a possible association between drug choices and the pattern of antihypertensive and anti-inflammatory responses, differences between users and nonusers of the therapeutic classes were investigated (Table 2). This analysis revealed that all baseline characteristics, such as clinical and biochemical parameters, remained well matched between groups and that the main intervention regimens employed could produce very similar changes in mean BP levels, with average systolic BP, which was moderately hypertensive (160-179 $\mathrm{mmHg}$ ) at baseline, dropping to a mild hypertensive state $(140-159 \mathrm{mmHg})^{16}$ in all therapeutic scenarios. Furthermore, this approach revealed that only users of RAS antagonists and users of diuretics exhibited a magnitude of treatment-induced reduction in circulating IL-6 and TNF- $\alpha$ similar to that observed in the whole group, whereas treatment with blockers of either calcium-channel or $\beta$-adrenergic receptors failed to parallel the general trend. Differences in IFN- $\gamma$ levels remained not apparent in spite of this analytical step.

\section{Discussion}

In order to assess whether a circulating cytokine-reducing effect was likely to result from hypertension management practices in current clinical practice, the present study investigated a possible anti-inflammatory response in patients receiving different drug prescriptions within the norms of a nationwide (Brazilian) consensus on treatment of systemic arterial hypertension. In this study, the pattern of drug usage achieved was consistent with the routine on hypertension management that is carried out by medical practitioners nationally, ${ }^{20}$ with a trend toward reproducing the medical attention that is directed to hypertensive older adults in the
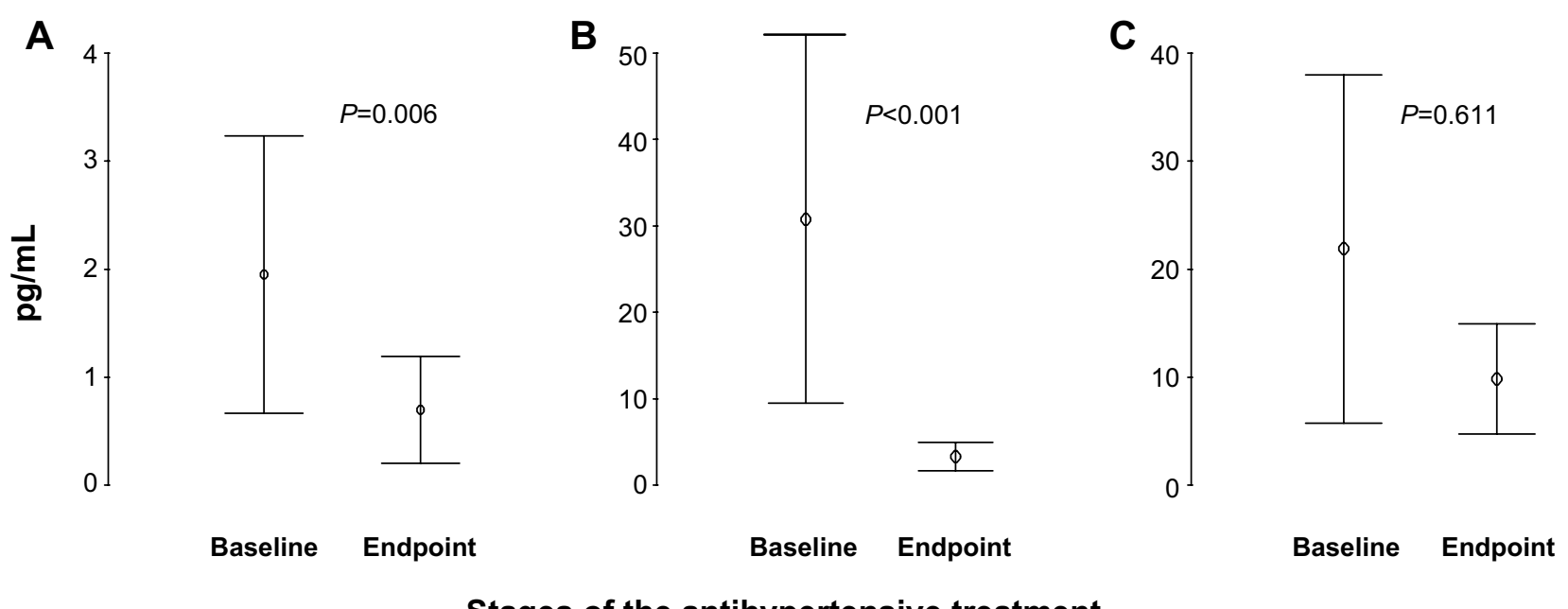

\section{Stages of the antihypertensive treatment}

Figure I General effects of customized antihypertensive drug therapy on circulating cytokine levels in a whole-group analysis ( $\mathrm{n}=\mathrm{I}$ I0). Notes: (A) IL-6. (B) TNF- $\alpha$. (C) IFN- $\gamma$. Data are expressed as means \pm standard error. $P$-values from two-related-samples Wilcoxon test. Abbreviations: IFN, interferon; IL, interleukin; TNF, tumor necrosis factor. 
Table 2 Effects of customized antihypertensive drug therapy on circulating levels of IL-6, TNF- $\alpha$, and IFN- $\gamma$ comparing users and nonusers of the most common antihypertensive classes

\begin{tabular}{|c|c|c|c|c|c|c|c|}
\hline \multirow[t]{2}{*}{ Drug class } & \multirow[t]{2}{*}{ Condition (n) } & \multicolumn{2}{|c|}{ IL-6 (pg/mL) } & \multicolumn{2}{|c|}{ TNF- $\alpha(p g / m L)$} & \multicolumn{2}{|c|}{ IFN- $\gamma(\mathrm{pg} / \mathrm{mL})$} \\
\hline & & Baseline & Endpoint & Baseline & Endpoint & Baseline & Endpoint \\
\hline \multirow[t]{2}{*}{ RAS antagonists } & Users (6I) & $1.6 \pm 0.5$ & $1.0 \pm 0.4$ & $21.1 \pm 9.9$ & $4.3 \pm 1.3^{*}$ & $16.5 \pm 7.8$ & $14.9 \pm 4.3$ \\
\hline & Nonusers (49) & $2.4 \pm 1.3$ & $0.3 \pm 0.2^{\#}$ & $42.8 \pm 20.5$ & $2.1 \pm 0.9 *$ & $28.5 \pm 15.3$ & $3.5 \pm 1.7$ \\
\hline \multirow[t]{2}{*}{ Diuretics } & Users (70) & $2.6 \pm 1.0$ & $0.9 \pm 0.4^{*}$ & $40.7 \pm 16.6$ & $4.2 \pm 1.2 *$ & $22.8 \pm 10.8$ & $7.3 \pm 3.2$ \\
\hline & Nonusers (40) & $0.7 \pm 0.2$ & $0.3 \pm 0.2$ & $13.4 \pm 3.5$ & $1.9 \pm 0.9 \ddagger$ & $20.2 \pm 11.7$ & $|4.2 \pm 4|$. \\
\hline \multirow[t]{2}{*}{$\mathrm{Ca}^{2+}$ channel blockers } & Users (27) & $1.5 \pm 0.7$ & $0.6 \pm 0.4$ & $36.4 \pm 30.3$ & $1.6 \pm 1.3$ & $1.2 \pm 0.7$ & $5.0 \pm 2.7$ \\
\hline & Nonusers (83) & $2.1 \pm 0.8$ & $0.7 \pm 0.3 *$ & $28.9 \pm 10.2$ & $3.9 \pm 1.0^{\#}$ & $28.6 \pm 10.6$ & $11.4 \pm 3.2$ \\
\hline \multirow[t]{2}{*}{$\beta$-adrenergic blockers } & Users (22) & $0.6 \pm 0.3$ & $1.0 \pm 0.6$ & $11.3 \pm 4.2$ & $6.0 \pm 2.7$ & $43.9 \pm 27.7$ & $16.2 \pm 9.7$ \\
\hline & Nonusers (88) & $2.3 \pm 0.8$ & $0.6 \pm 0.3 *$ & $35.6 \pm 13.7$ & $2.7 \pm 0.8^{\ddagger}$ & $16.4 \pm 7.3$ & $8.2 \pm 2.1$ \\
\hline
\end{tabular}

Notes: Data expressed as means \pm standard error. $P$-values from two-related-samples Wilcoxon test. $* P<0.05 ; \# P<0.005$; $¥ P<0.001$.

Abbreviations: IFN, interferon; IL, interleukin; n, number; RAS, renin-angiotensin system; TNF, tumor necrosis factor.

Unified Health System (SUS - Sistema Único de Saúde), the Brazilian public health care service that is characterized by insufficient assistance to all citizens despite the premise of universal coverage. ${ }^{21}$ Using this protocol, we demonstrated that short-term, clinically guided antihypertensive treatment is effective in reversing the low-grade proinflammatory state of serum cytokines found in older women with different metabolic disorders, known as "inflammaging." 14 The most interesting result obtained in our analysis was the absence of a cytokine-lowering effect with the use of $\beta$-adrenoceptor blockers, despite experimental evidence in favor of such action. Taken together, our results provide evidence that the pharmaceutical classes of $\mathrm{Ca}^{2+}$ channel and $\beta$-adrenergic blockers per se do not produce an anti-inflammatory response. But our findings do not rule out anti-inflammatory properties of these drug classes by means other than a cytokine-lowering effect, or even by this very mechanism but not in a form as intense as that induced by RAS antagonists and diuretics under field conditions.

Diuretics, which were not expected to reduce cytokines, produced a marked decrease in serum levels of both IL- 6 and TNF- $\alpha$. Our results are not likely to derive from an overlap between the use of diuretics and RAS antagonists, since concomitant prescription of both classes was observed in only $28.8 \%(n=31)$ of all cases. Based on chi-square analyses, we concluded that both drug classes had a dissimilar, excluding distribution among the older women, since $79.6 \%$ of the nonusers of RAS antagonists were users of diuretics, and $75 \%$ of the nonusers of diuretics were users of RAS antagonists $\left(\chi^{2}=9.72 ; P=0.002\right)$. This counterintuitive effect of diuretics is not entirely surprising, as our data corroborate the small amount of literature evidence in which a loop diuretic as well as a potassium-sparing counterpart attenuated levels of the same proinflammatory cytokines considered herein. ${ }^{22-26}$
Although the exact anti-inflammatory mechanism of diuretics remains unknown, our data support extracardiac benefits, as all options of drug therapy used in this study were associated with a significant lowering in both systolic and diastolic BP, which, if effective per se, would have produced a similar cytokine-lowering response by treatment with all other drug classes. Therefore, reduction in IL- 6 and TNF- $\alpha$ levels may not be related solely to biomechanical relief of peripheral baroreceptors as described elsewhere for IFN- $\gamma$ levels. ${ }^{27}$

An intriguing result was a nonsignificant effect of the treatment with RAS antagonists on serum IL-6 levels. Although epidemiological studies have reported an inverse correlation between IL- 6 and successful aging, ${ }^{28,29}$ IL-6 is likely to act as a surrogate marker of TNF- $\alpha$, because the production of the two cytokines is closely related (TNF- $\alpha$ triggers IL- 6 production $)^{30}$ and also because local TNF- $\alpha$ does not readily escape through the bloodstream and only gradually rises with the progression of pathological aging. ${ }^{31,32}$ Moreover, although IL-6 is often classified as proinflammatory, it also has very important, context-sensitive antiinflammatory properties, including inhibition of TNF- $\alpha$ gene transcription, ${ }^{33}$ which are more prominent in the absence of TNF- $\alpha$ expression. ${ }^{34}$

The present study had some limitations. Firstly, timing (number and frequency of appointments) and dosage (amount and frequency of drug consumption) of any drug intervention may have a major impact on determining acute inflammatory responses, and the present analyses were not controlled for such variables. Thus, these interferences cannot be ruled out. Secondly, the rather small number of subjects, mainly in analyses involving segregation, may have limited the ability to determine differences in biomarkers and clinical outcomes. Thirdly, since the study was conducted with a series of female patients, the authors do 
not suggest direct extrapolation of our conclusions to male subjects due to substantial sex-related changes in basal and stimulated cytokine levels. ${ }^{35,36}$ At last, because our design aimed at evaluating short-term response to antihypertensive pharmacotherapy, interventions were performed based on the assumption that most treatments would not achieve optimal endpoints, which may have limited the extent of the cytokine-lowering effect observed in our setting. Nonetheless, the authors believe that the study reproduces the current scenario of hypertension management practices in our country and abroad, where suboptimal control is often the best outcome that is possible.

\section{Conclusion}

Our study revealed that attaining to the antihypertensive drug therapy advocated by the Brazilian guidelines significantly attenuates serum levels of the most relevant proinflammatory mediators implicated in the inflammaging of the human biology, with undetectable effects on other mediators involved to a lesser extent in the phenomenon. These data reassure extracardiac, anti-inflammatory benefits from BP-lowering drug therapy in the elderly. Our findings also suggest that the RAS is likely to play an important role in this overstimulated state of the aging immune system and encourage further investigation on the pathway as well as on possible modulators of the inflammaging, particularly in a population with high prevalence of metabolic syndrome. ${ }^{37} \mathrm{~A}$ body of data has shown that even after normalization of BP, hypertensive individuals continue to have an enhanced risk of coronary events as compared with nonhypertensive ones. ${ }^{38,39}$ Equivalent BP control by different therapeutic regimens has resulted in distinct incidence of cardiovascular events and complications. ${ }^{40,41}$ Evidence such as this has reinforced the concept that high BP is indeed only an element of the hypertensive syndrome.

By both hemodynamic and molecular mechanisms, hypertension is strongly associated with systemic inflammatory activity. This effect has an important role in atherogenesis, and may be a link to the persistent high risk for cardiovascular events in hypertensive individuals under effective BP control. To the present, however, very little is known about the impact of different antihypertensive therapies on systemic inflammatory activity. In this context, the objective of this study was to compare in a "real life" setting the anti-inflammatory effect of different antihypertensive treatments. Our findings open the door to discussion of how different antihypertensive treatments may be, even when they obtain similar control of BP. But because association does not necessarily indicate causation, and to determine if the association observed herein is other than incidental, such relationship must be tested using other study designs, such as public policy-guided interventions abroad and/or with larger samples, as long as comparable age and sex strata and drug therapy options are considered. The effects of other candidate mediators and associated factors should also be tested in these patients.

\section{Acknowledgments}

This work was supported by Conselho Nacional de Desenvolvimento Científico e Tecnológico (CNPq) (grant \# 484318/2006-3) and Decanato de Pesquisa e Pós-Graduação/ University of Brasília (grant \# 100650/2009). VC Sousa and AC Tonet-Furioso received student fellowships from Coordenação de Aperfeiçoamento de Pessoal de Nível Superior and CNPq, respectively. LCC Afonso and OT Nóbrega received fellowships for productivity in research from CNPq.

\section{Disclosure}

The authors report no conflicts of interest in this work.

\section{References}

1. Cheng ZJ, Vapaatalo H, Mervaala E. Angiotensin II and vascular inflammation. Med Sci Monit. 2005;11(6):RA194-RA205.

2. Suzuki Y, Ruiz-Ortega M, Gomez-Guerrero C, Tomino Y, Egido J. Angiotensin II, the immune system and renal diseases: another road for RAS? Nephrol Dial Transplant. 2003;18(8):1423-1426.

3. Gage JR, Fonarow G, Hamilton M, Widawski M, Martínez-Maza O, Vredevoe DL. Beta blocker and angiotensin-converting enzyme inhibitor therapy is associated with decreased $\mathrm{Th} 1 / \mathrm{Th} 2$ cytokine ratios and inflammatory cytokine production in patients with chronic heart failure. Neuroimmunomodulation. 2004;11(3):173-180.

4. Rosenson RS. Modulating atherosclerosis through inhibition or blockade of angiotensin. Clin Cardiol. 2003;26(7):305-311.

5. Rosenson RS. New approaches in the intensive management of cardiovascular risk in the metabolic syndrome. Curr Probl Cardiol. 2005;30(5):241-279.

6. Steen V. Targeted therapy for systemic sclerosis. Autoimmun Rev. 2006; 5(2):122-124.

7. Zhang X, Li ZZ, Liu DF, Xu X, Mei ZC, Shen W. Angiotensinconverting enzyme inhibitors improve hepatic steatosis by modulating expression of tumour necrosis factor-alpha, interleukin- 6 and adiponectin receptor-2 in rats with type 2 diabetes. Clin Exp Pharmacol Physiol. 2009;36(7):631-636.

8. Hagiwara S, Iwasaka H, Matumoto S, Hidaka S, Noguchi T. Effects of an angiotensin-converting enzyme inhibitor on the inflammatory response in in vivo and in vitro models. Crit Care Med. 2009;37(2):626-633.

9. Flammer AJ, Sudano I, Hermann F, et al. Angiotensin-converting enzyme inhibition improves vascular function in rheumatoid arthritis. Circulation. 2008;117(17):2262-2269.

10. Sanz-Rosa D, Oubiña MP, Cediel E, et al. Effect of AT1 receptor antagonism on vascular and circulating inflammatory mediators in SHR: role of NF-kappaB/IkappaB system. Am J Physiol Heart Circ Physiol. 2005;288(1):H111-H115. 
11. Verhoeckx KC, Gaspari M, Bijlsma S, et al. In search of secreted protein biomarkers for the anti-inflammatory effect of beta2-adrenergic receptor agonists: application of DIGE technology in combination with multivariate and univariate data analysis tools. J Proteome Res. 2005;4(6):2015-2023.

12. Izeboud CA, Monshouwer M, van Miert AS, Witkamp RF. The betaadrenoceptor agonist clenbuterol is a potent inhibitor of the LPS-induced production of TNF-alpha and IL-6 in vitro and in vivo. Inflamm Res. 1999;48(9):497-502.

13. Ackland GL, Yao ST, Rudiger A, et al. Cardioprotection, attenuated systemic inflammation, and survival benefit of beta1-adrenoceptor blockade in severe sepsis in rats. Crit Care Med. 2010;38(2):388-394.

14. Franceschi C, Capri M, Monti D, et al. Inflammaging and anti-inflammaging: a systemic perspective on aging and longevity emerged from studies in humans. Mech Ageing Dev. 2007;128(1):92-105.

15. Moraes CF, Souza ER, Souza VC, et al. A common polymorphism in the renin angiotensin system is associated with differential outcome of antihypertensive pharmacotherapy prescribed to Brazilian older women. Clin Chim Acta. 2008;396(1-2):70-75.

16. IV Brazilian Guidelines in Arterial Hypertension Work Groups [IV Brazilian guidelines in arterial hypertension]. Arq Bras Cardiol. 2004; 82 Suppl 4:7-22. Portuguese.

17. Cockcroft DW, Gault MH. Prediction of creatinine clearance from serum creatinine. Nephron. 1976;16(1):31-41.

18. Friedewald WT, Levy RI, Fredrickson DS. Estimation of the concentration of low-density lipoprotein cholesterol in plasma, without use of the preparative ultracentrifuge. Clin Chem. 1972;18(6):499-502.

19. International Diabetes Federation. IDF Global Guideline for Managing Older People with Type 2 Diabetes. Available from: http://www.idf.org/ sites/default/files/IDF-Guideline-for-older-people-T2D.pdf. Accessed December 11, 2014.

20. Mion D Jr, da Silva GV, de Gusmão JL, et al. Do Brazilian physicians follow the Brazilian guidelines on hypertension? Arq Bras Cardiol. 2007;88(2):212-217.

21. Nóbrega OT, Faleiros VP, Telles JL. Gerontology in the developing Brazil: achievements and challenges in public policies. Geriatr Gerontol Int. 2009;9(2):135-139.

22. Yuengsrigul A, Chin TW, Nussbaum E. Immunosuppressive and cytotoxic effects of furosemide on human peripheral blood mononuclear cells. Ann Allergy Asthma Immunol. 1999;83(6 Pt 1):559-566.

23. Xu B, Makris A, Thornton C, Ogle R, Horvath JS, Hennessy A. Antihypertensive drugs clonidine, diazoxide, hydralazine and furosemide regulate the production of cytokines by placentas and peripheral blood mononuclear cells in normal pregnancy. J Hypertens. 2006; 24(5):915-922.

24. Rolfe MW, Kunkel SL, Rowens B, Standiford TJ, Cragoe EJ Jr, Strieter RM. Suppression of human alveolar macrophage-derived cytokines by amiloride. Am J Respir Cell Mol Biol. 1992;6(6):576-582.

25. Haddad JJ, Land SC. Amiloride blockades lipopolysaccharideinduced proinflammatory cytokine biosynthesis in an IkappaB-alpha/ NF-kappaB-dependent mechanism. Evidence for the amplification of an antiinflammatory pathway in the alveolar epithelium. Am J Respir Cell Mol Biol. 2002;26(1):114-126.
26. Haddad JJ. Amiloride and the regulation of NF-kappaB: an unsung crosstalk and missing link between fluid dynamics and oxidative stressrelated inflammation - controversy or pseudo-controversy? Biochem Biophys Res Commun. 2005;327(2):373-381.

27. Gavrilova SA, Demidov LV, Medvedeva NA, Ashmarin IP. Chronic administration of interferon-a decreases blood pressure and heart rate in rats. Bull Exp Biol Med. 2000;129(5):413-416.

28. Forsey RJ, Thompson JM, Ernerudh J, et al. Plasma cytokine profiles in elderly humans. Mech Ageing Dev. 2003;124(4):487-493.

29. Maggio M, Guralnik JM, Longo DL, Ferrucci L. Interleukin-6 in aging and chronic disease: a magnificent pathway. J Gerontol A Biol Sci Med Sci. 2006;61(6):575-584.

30. Delves PJ, Roitt IM. The immune system. Second of two parts. NEngl J Med. 2000;343(2):108-117.

31. Roubenoff R, Harris TB, Abad LW, Wilson PW, Dallal GE, Dinarello CA. Monocyte cytokine production in an elderly population: effect of age and inflammation. J Gerontol A Biol Sci Med Sci. 1998;53(1): M20-M26.

32. Krabbe KS, Pedersen M, Bruunsgaard H. Inflammatory mediators in the elderly. Exp Gerontol. 2004;39(5):687-699.

33. Starkie R, Ostrowski SR, Jauffred S, Febbraio M, Pedersen BK. Exercise and IL-6 infusion inhibit endotoxin-induced TNF-alpha production in humans. FASEB J. 2003;17(8):884-886.

34. Petersen AM, Pedersen BK. The anti-inflammatory effect of exercise. J Appl Physiol (1985). 2005;98(4):1154-1162.

35. Bruunsgaard H, Pedersen AN, Schroll M, Skinhoj P, Pedersen BK Impaired production of proinflammatory cytokines in response to lipopolysaccharide (LPS) stimulation in elderly humans. Clin Exp Immunol. 1999;118(2):235-241.

36. Bonafè M, Olivieri F, Cavallone L, et al. A gender - dependent genetic predisposition to produce high levels of IL-6 is detrimental for longevity. Eur J Immunol. 2001;31(8):2357-2361.

37. Karnikowski M, Córdova C, Oliveira RJ, Karnikowski MG, Nóbrega Ode T. Non-alcoholic fatty liver disease and metabolic syndrome in Brazilian middle-aged and older adults. Sao Paulo Med J. 2007;125(6):333-337.

38. Epstein BJ, Smith SM, Choksi R. Recent changes in the landscape of combination RAS blockade. Expert Rev Cardiovasc Ther. 2009;7(11): 1373-1384.

39. JATOS Study Group. Principal results of the Japanese trial to assess optimal systolic blood pressure in elderly hypertensive patients (JATOS). Hypertens Res. 2008;31(12):2115-2127.

40. Saruta T, Hayashi K, Ogihara T, Nakao K, Fukui T, Fukiyama K; CASE-J Study Group. Effects of candesartan and amlodipine on cardiovascular events in hypertensive patients with chronic kidney disease: subanalysis of the CASE-J Study. Hypertens Res. 2009;32(6):505-512.

41. Ogihara T, Fujimoto A, Nakao K, Saruta T; CASE-J Trial Group. ARB candesartan and CCB amlodipine in hypertensive patients: the CASE-J trial. Expert Rev Cardiovasc Ther. 2008;6(9):1195-1201.
Clinical Interventions in Aging

\section{Publish your work in this journal}

Clinical Interventions in Aging is an international, peer-reviewed journal focusing on evidence-based reports on the value or lack thereof of treatments intended to prevent or delay the onset of maladaptive correlates of aging in human beings. This journal is indexed on PubMed Central, MedLine,

\section{Dovepress}

CAS, Scopus and the Elsevier Bibliographic databases. The manuscript management system is completely online and includes a very quick and fair peer-review system, which is all easy to use. Visit http://www.dovepress. com/testimonials.php to read real quotes from published authors. 